\title{
Consumo no Brasil: Comportamento Otimizador, Restrição de Crédito ou Miopia?*
}

\author{
Fábio Augusto Reis Gomes ${ }^{\dagger}$
}

\footnotetext{
Conteúdo: 1. Introdução; 2. Teoria de Consumo; 3. Metodologia Econométrica; 4. Conclusão; A. Apêndice.

Palavras-chave: Consumo; Comportamento Otimizador; Miopia; Restrição de Crédito.

Códigos JEL: C22; E21.
}

Este artigo analisa a evolução do consumo no Brasil usando um arcabouço que comporta três hipóteses distintas: comportamento otimizador, miopia e restrição de crédito. Os resultados econométricos sugerem que os juros não são significativos, o que rejeita à hipótese de comportamento otimizador. Por outro lado, os resultados não são capazes de confirmar a hipótese de miopia nem tão pouco a de restrição de crédito. Não obstante, pode-se observar que, em alguns anos, há suavização de consumo, pois as taxas de crescimento do consumo e da renda têm sinais opostos. Sendo assim, tal observação é incompatível com a hipótese de miopia.

This paper analyzes the evolution of Brazilian consumption taking into account three different theories: optimization behavior, myopia and credit constraint. The econometric results suggest that interest rates are irrelevant, which goes against what is predicted by the optimization behavior hypothesis. On the other side, the results are not able to confirm neither myopia nor credit constraint. In spite of that, it is possible to observe that, in some years, consumption is smoothed because its growth rate and the income growth rate have opposite signs. Thus, this fact is incompatible with the myopic behavior.

\footnotetext{
*O autor agradece a Ari Francisco de Araújo Júnior, Cleomar Gomes, Eduardo Giannetti da Fonseca, Humberto Spolador, ao editor Arilton Teixeira e aos pareceristas anônimos pelos comentários e ao assistente Fábio Luiz Alves de Andrade Santos por todo auxílio, isentando-os de quaisquer erros remanescentes. $O$ autor agradece ao CNPp pelo apoio a este projeto.

†Insper Instituto de Ensino e Pesquisa. Rua Quatá, 300, $4^{\circ}$ Andar, Sala 422, Vila Olímpia. São Paulo, SP, Brasil - CEP: $04546-042$. Tel.: (55) 11 4504-2776. E-mail: FabioARG@insper.org.br
} 


\section{INTRODUÇÃO}

O objetivo deste artigo é confrontar três diferentes teorias sobre a decisão de consumo. A primeira supõe que os indivíduos têm um comportamento otimizador e, por conseguinte, o consumo obedeceria à Equação de Euler, proveniente do problema intertemporal do consumidor, sendo uma função da taxa de juros. Esta teoria pode ser vista como uma versão da Teoria da Renda Permanente (TRP), devida a Friedman (1957), já que ambas sugerem que o consumidor suaviza seu consumo ao longo da vida. A segunda teoria argumenta que parte dos consumidores está restrita ao crédito e, por isso, segue uma regra de bolso: consumir sua renda corrente ao invés da renda permanente (Campbell e Mankiw, 1989). Porém, Shea (1995) argumenta que os consumidores seguem tal regra de bolso apenas se eles são míopes. Segundo esse autor, quando os consumidores não têm acesso ao crédito, há um impedimento para se conseguir um empréstimo, mas não para poupar. Logo, a taxa de crescimento do consumo deve apresentar uma correlação maior com a taxa de crescimento da renda em períodos nos quais há um crescimento da renda. Em outras palavras, se a renda diminui, o consumidor pode utilizar seus recursos acumulados para suavizar o consumo. Shea (1995) argumenta, então, que reações simétricas do consumo à renda constituem-se em uma evidência favorável à hipótese de miopia enquanto reações assimétricas favorecem a hipótese de restrição de crédito. ${ }^{1}$

A literatura nacional sobre decisão de consumo cresceu bastante recentemente, destacando-se Reis et alii (1998), Issler e Rocha (2000), Gomes (2004), Gomes e Paz (2004) e Gomes et alii (2005). No entanto, nenhum destes estudos explorou a distinção entre restrição de crédito e miopia, como sugerido por Shea (1995). Logo, tal investigação constitui-se na maior contribuição deste artigo.

Para o caso brasileiro as únicas séries longas de consumo e renda são anuais e datam de 1947 a 2005. As séries de consumo agregam tanto consumo de bens não-duráveis quanto de bens duráveis, uma característica indesejada, uma vez que, em princípio, ambos possuem comportamentos distintos. Contudo, utilizando as séries de consumo de bens duráveis e bens não duráveis construídas por Ellery Jr. et alii (2002) para o período de 1970 a 1998, Gomes et alii (2005) não rejeitam a hipótese de separabilidade nas decisões de consumo destes dois tipos de bens. Além disso, as evidências desses autores sugerem que o consumo de duráveis e não-duráveis, além da renda, possuem comovimento de longo prazo (cointegração) e curto prazo (ciclos comuns). Se por um lado estes resultados amenizam os problemas causados pela agregação destas séries, é importante ter em mente que a própria análise de Gomes et alii (2005) deve ser vista com cautela. A limitada disponibilidade de dados pode ter impactado o poder dos testes utilizados, inclusive o de separabilidade na decisão de consumo. De todo modo, este estudo utiliza as séries de 1947 a 2005, o que é particularmente importante para se garantir que a amostra apresente um número razoável de períodos nos quais a taxa de crescimento da renda é negativa. ${ }^{2}$

Os principais resultados obtidos são estes. Primeiro, considerando o modelo proposto por Campbell e Mankiw (1989) são encontrados resultados similares aos da literatura nacional: o consumo é sensível a variações da renda, mas não parece responder aos juros. Segundo, ao considerar o modelo de Shea (1995) a taxa de juros é, em geral, não significativa. Porém, os modelos econométricos não são capazes de discernir se este resultado se deve à hipótese de miopia ou a de restrição de crédito. A despeito desta indecisão, observamos que em alguns anos as taxas de crescimento do consumo e da renda apresentam sinais opostos, o que é uma evidência à favor de suavização de consumo e contrária à hipótese de miopia. Nos anos de 1982, 1990 e 1991 ocorreu um crescimento do consumo mesmo com a retração da renda; já nos anos de 1953, 1975, 1987, 1989 e 2002 apesar da renda apresentar uma taxa de crescimento positiva, o oposto ocorreu com o consumo. Esta suavização é restrita a poucos anos, o que é compatível apenas com a hipótese de restrição de crédito.

\footnotetext{
${ }^{1}$ Esta assimetria no comportamento do consumo foi ressaltada primeiramente por Altonji e Siow (1987).

${ }^{2}$ Aproximadamente $25 \%$ dos anos da amostra utilizada neste estudo apresenta uma taxa de crescimento da renda negativa enquanto no estudo de Shea (1995) este percentual não atinge $15 \%$.
} 
Além desta Introdução o artigo é organizado da seguinte forma. A Seção 2 apresenta uma breve revisão dos modelos teóricos, bem como a derivação das equações de teste. A Seção 3 apresenta a base de dados e os resultados econométricos. A última seção apresenta as Conclusões.

\section{TEORIA DE CONSUMO}

\subsection{Comportamento otimizador}

Como discutido por Hall (1978), o problema intertemporal do consumidor representativo fowardlooking é maximizar seu bem-estar ao longo da vida, escolhendo sua trajetória de consumo, $c_{t}$, sujeito a uma restrição orçamentária. A solução deste problema é caracterizada pela Equação de Euler:

$$
u^{\prime}\left(c_{t}\right)=E_{t}\left[\beta u^{\prime}\left(c_{t+1}\right)\left(1+r_{t+1}\right)\right]
$$

em que $u^{\prime}(\cdot)$ é a utilidade marginal do consumo, $\beta$ é a taxa de desconto intertemporal, $r_{t+1}$ é a taxa de retorno real da riqueza do período $t$ para o período $t+1$ e $E_{t}$ é a esperança condicional ao conjunto de informação disponível no período $t, I_{t}{ }^{3}$ Considerando a função utilidade CRRA,

$$
u(c)=\frac{c^{1-\sigma}}{1-\sigma}
$$

em que $\sigma$ é a aversão relativa ao risco, a Equação de Euler (1) torna-se:

$$
E_{t}\left[\left(\frac{c_{t+1}}{c_{t}}\right)^{-\sigma}\left(1+r_{t+1}\right)\right]=\frac{1}{\beta}
$$

Para estimar esta equação autores como Mankiw (1981), Hansen e Singleton (1983) e Hall (1988) consideram sua aproximação linear de primeira ordem, ${ }^{4}$ segundo a qual:

$$
\Delta \ln \left(c_{t+1}\right)=\mu+\frac{1}{\sigma} r_{t+1}+\varepsilon_{t+1}
$$

Utilizando dados da economia americana, Mankiw (1981) conclui que a equação (3) não é capaz de explicar toda a correlação serial dos dados. Hansen e Singleton (1983) não obtêm estimativas significativas da aversão ao risco. Hall (1988) conclui que a elasticidade de substituição intertemporal, $1 / \sigma$, é próxima de zero, cuja implicação é uma taxa de crescimento do consumo pouco sensível à taxa de juros. Os resultados são similares na literatura nacional. Por exemplo, Reis et alii (1998) também concluem que a resposta da taxa de crescimento do consumo à taxa de juros é não significativa. Uma primeira explicação para o baixo poder explicativo da equação (3) é a seguinte: ao modelar os gastos de bens não-duráveis em separado dos gastos de bens duráveis, implicitamente, uma hipótese de separabilidade na função de utilidade é adotada. ${ }^{5}$ Logo, se tal hipótese é demasiadamente restritiva, sua adoção poderia explicar a performance ruim do modelo. Utilizando as séries de consumo de bens duráveis e não-duráveis de 1970 a 1998 construídas por Ellery Jr. et alii (2002), Gomes et alii (2005) investigam esta

\footnotetext{
${ }^{3}$ Sob certas hipóteses, a solução deste problema é equivalente a Teoria da Renda Permanente (TRP), segundo a qual o indivíduo consume sua renda permanente. Hall (1978) assume uma utilidade quadrática e que $\beta\left(1+r_{t+1}\right)=1$, obtendo a hipótese do passeio aleatório do consumo. Flavin (1981) considera explicitamente a TRP e chega a mesma conclusão.

${ }^{4}$ De fato, alguns autores assumem que o consumo e o juros têm uma distribuição conjunta log-normal com matriz de covariância constante ao longo do tempo. No entanto, isto é equivalente a uma expansão de Taylor de primeira ordem (ver apêndice).

${ }^{5}$ De forma análoga a Hall (1978), Mankiw (1982) analisa a decisão de consumo de bens duráveis, assumindo implicitamente a hipótese de separabilidade. Posteriormente, Bernanke (1985) modela conjuntamente a decisão de gastos em bens duráveis e não duráveis, sem supor separabilidade. Galí (1993) e Vahid e Engle (1997) também modelam conjuntamente a decisão de consumo de bens duráveis e não-duráveis, porém impondo a separabilidade $a$ priori.
} 
possibilidade para o caso de uma função utilidade CES e não rejeitam a hipótese de separabilidade nas decisões de consumo de duráveis e não-duráveis. Embora essa conclusão deva ser vista com cautela, dada a pequena disponibilidade de dados, para estender a equação (3) consideraremos às hipóteses de restrição de crédito e miopia.

\subsection{Restrição de crédito}

Motivados pelas falhas do modelo anterior, Campbell e Mankiw (1989) sugerem que as evidências empíricas dos Estados Unidos são melhor explicadas quando são considerados dois tipos de agentes: um otimizador que consome segundo a equação (3) e outro que usa a regra de bolso de consumir sua renda corrente, devido à falta de acesso ao crédito. Para chegar a tal conclusão, os autores utilizam a seguinte equação de teste:

$$
\Delta \ln \left(c_{t+1}\right)=\lambda \Delta \ln \left(y_{t+1}\right)+(1-\lambda)\left[\mu+\frac{1}{\sigma} r_{t+1}+\varepsilon_{t+1}\right]
$$

em que $\lambda$ é a fração da renda agregada que pertence a consumidores restritos ao crédito. Se $\lambda=1$, os consumidores não têm acesso ao crédito, estando fadados a consumir a renda corrente. Por conseguinte, a taxa de crescimento do consumo depende apenas da taxa de crescimento da renda corrente. Por outro lado, caso $\lambda=0$, haveria evidência de que os consumidores não estão restritos ao crédito, podendo suavizar seu consumo ao longo da vida. ${ }^{6}$

Vários autores estimaram este modelo para o caso brasileiro. Utilizando séries de consumo que agregam tanto bens duráveis quanto bens não-duráveis Cavalcanti (1993) estima $\lambda$ em torno de 0,32 , Reis et alii (1998) encontram evidência de que $\lambda$ é, aproximadamente, igual a 0,80 , enquanto Issler e Rocha (2000), Gomes e Paz (2004) e Gomes (2004) obtêm, em média, 0,74, 0,61 e 0,85, respectivamente. Utilizando uma série de consumo de bens duráveis, Gomes et alii (2005) também encontram ampla evidência de restrição de crédito. Note que tal fato explicaria a baixa sensibilidade do consumo à taxa de juros, pois quanto maior $\lambda$, menor é o coeficiente da taxa de juros na equação (4).

\subsection{Miopia}

Shea (1995) argumenta que o modelo de Campbell e Mankiw (1989) refere-se ao caso de miopia, pois apenas agentes míopes reagiriam de forma idêntica a aumentos ou reduções na renda. Sob restrição de crédito, os agentes não podem tomar emprestado, mas podem acumular riqueza e, como resultado, o consumo deveria responder mais fortemente à taxa de crescimento da renda quando esta é positiva. Para testar esta conjectura, Shea (1995) utilizou a seguinte equação de teste:

$$
\Delta \ln \left(c_{t+1}\right)=\pi_{0}+\pi_{1} I_{p} \Delta \ln \left(y_{t+1}\right)+\pi_{2} I_{n} \Delta \ln \left(y_{t+1}\right)+\pi_{3} r_{t+1}+\varepsilon_{t+1}
$$

em que $I_{p}=1$ se $\Delta \ln \left(y_{t+1}\right)>0$ e zero caso contrário; $I_{n}=1-I_{p}$. Se os consumidores não são míopes, nem tão pouco restritos ao crédito, a taxa de crescimento do consumo depende apenas da taxa de juros $\left(\pi_{1}=\pi_{2}=0\right)$. Se os consumidores são míopes, mudanças na renda corrente são transmitidas imediatamente para o consumo e, por conseguinte, $\pi_{1}$ e $\pi_{2}$ devem ser significativos, positivos e iguais.

\footnotetext{
${ }^{6}$ Como a equação (4) é estimada com um estimador de variáveis instrumentais e os instrumentos são variáveis defasadas, o regressor é o valor previsto da taxa de crescimento da renda, $E_{t} \Delta \ln \left(y_{t+1}\right)$. Sob a TRP espera-se $\lambda=0$, porque toda informação previsível já foi incorporada à renda permanente e, por conseguinte, ao consumo, não podendo haver nenhum efeito adicional sobre o mesmo. Em outras palavras, como a inovação na renda corrente é correlacionada com a inovação na renda permanente, mesmo sob a validade da TRP, $\Delta \ln \left(y_{t+1}\right)$ pode ter um impacto significativo sobre $\Delta \ln \left(c_{t+1}\right)$. Por isso, é necessário usar o valor previsto de $\Delta \ln \left(y_{t+1}\right)$. Esta ressalva é válida para todas as equações de teste utilizadas e justifica o uso de variáveis instrumentais.
} 
Sob restrição de crédito, $\pi_{1}$ deve ser significativo e maior do que $\pi_{2}$. Nos dois últimos casos espera-se que a taxa de juros seja não significativa $\left(\pi_{3}=0\right)$.

Estas restrições podem ser entendidas de forma alternativa definindo-se $\lambda_{1}, \lambda_{2}$ e $\lambda_{3}=1-\lambda_{1}-\lambda_{2}$ como a proporção de indivíduos restritos ao crédito, míopes e otimizadores, respectivamente. Então, agregando os diferentes tipos de consumidores obtemos:?

$$
\Delta \ln \left(c_{t+1}\right)=\lambda_{1}\left[\delta_{p} I_{p} \Delta \ln \left(y_{t+1}\right)+\delta_{n} I_{n} \Delta \ln \left(y_{t+1}\right)\right]+\lambda_{2} \Delta \ln \left(y_{t+1}\right)+\lambda_{3}\left[\mu+\frac{1}{\sigma} r_{t+1}+\varepsilon_{t+1}\right]
$$

em que $\delta_{p}$ é positivo e maior do que $\delta_{n}$. Como $\Delta \ln \left(y_{t+1}\right)=I_{p} \Delta \ln \left(y_{t+1}\right)+I_{n} \Delta \ln \left(y_{t+1}\right)$, conclui-se que:

$$
\Delta \ln \left(c_{t+1}\right)=\underbrace{\lambda_{3} \mu}_{\pi_{0}}+\underbrace{\left(\lambda_{1} \delta_{p}+\lambda_{2}\right)}_{\pi_{1}} I_{p} \Delta \ln \left(y_{t+1}\right)+\underbrace{\left(\lambda_{1} \delta_{n}+\lambda_{2}\right)}_{\pi_{2}} I_{n} \Delta \ln \left(y_{t+1}\right)+\underbrace{\frac{\lambda_{3}}{\sigma}}_{\pi_{3}} r_{t+1}+\vartheta_{t+1}
$$

em que $\vartheta_{t+1}=\lambda_{3} \varepsilon_{t+1}$. Sob restrição de crédito, como $\delta_{p}>\delta_{n}$ espera-se $\pi_{1}>\pi_{2}$. No caso de ocorrer apenas miopia, espera-se $\pi_{1}=\pi_{2}=\lambda_{2}$. Além disso, como $\lambda_{3}=1-\lambda_{1}-\lambda_{2}$, quanto maior a evidência de miopia e/ou restrição de crédito, menor deve ser o impacto dos juros sobre a taxa de crescimento do consumo. Observe que, sem distinguir miopia de restrição de crédito, não é possível saber por que a taxa de juros é não significativa.

\section{METODOLOGIA ECONOMÉTRICA}

\subsection{Dados}

As séries utilizadas têm frequência anual, sendo considerados 2 períodos amostrais diferentes. 0 primeiro de 1947 a 2005, no qual estão disponíveis apenas as séries de consumo e renda, e o segundo de 1970 a 2005, no qual se encontram também disponíveis as séries de juros utilizadas. As séries de consumo e renda são o consumo final das famílias e a renda disponível bruta, respectivamente. Ambas foram deflacionadas pelo deflator implícito do PIB e divididas a cada ano pela população residente no Brasil no dia $1^{\circ}$ de julho para se obter séries reais per capita. ${ }^{8}$ Por fim, tomou-se o logaritmo natural de modo que $\ln \left(c_{t}\right)$ e $\ln \left(y_{t}\right)$ referem-se ao logarítimo natural do consumo real per capita e da renda real per capita, respectivamente. Na Figura 1, apresentamos estas séries.

Na segunda amostra foram consideradas duas taxas de juros: $C D B$ e poupança. Os retornos nominais em frequência anual foram deflacionados a partir do IGP-DI do seguinte modo,

$$
1+r_{t}=\frac{1+i_{t}}{1+\pi_{t}}
$$

em que $i_{t}$ é o CDB ou poupança anual nominal e $\pi_{t}$ é a inflação anual medida pelo IGP-DI. ${ }^{9}$ A Figura 2 apresenta as séries de CDB e poupança em termos reais.

\footnotetext{
${ }^{7}$ Esta equação pode ser vista como uma equação de aninhamento de modelos não aninhados.

${ }^{8}$ Todas estas séries foram extraídas do Ipeadata e tem como fonte o IBGE. O deflator implícito do PIB tem base em 2005 igual a 1.

${ }^{9} \mathrm{O}$ CDB foi extraído do Ipeadata e sua fonte é o Banco Central do Brasil. A poupança foi extraída da FGVDADOS tendo como fonte a ANDIMA. Ambos foram obtidos em frequência mensal (\% a.m.), sendo primeiramente acumulados dentro de cada ano. Com isso, foram obtidas taxas de juros anuais nominais. Utilizando o índice anual do IGP-DI foram construídas as séries reais. 0 IGP-DI tem como base 2005 igual a 1, sendo obtido do Ipeadata e sua fonte é FGV/Conjuntura Econômica.
} 
Figura 1: (ln) Consumo real per capita e (ln) renda real per capita

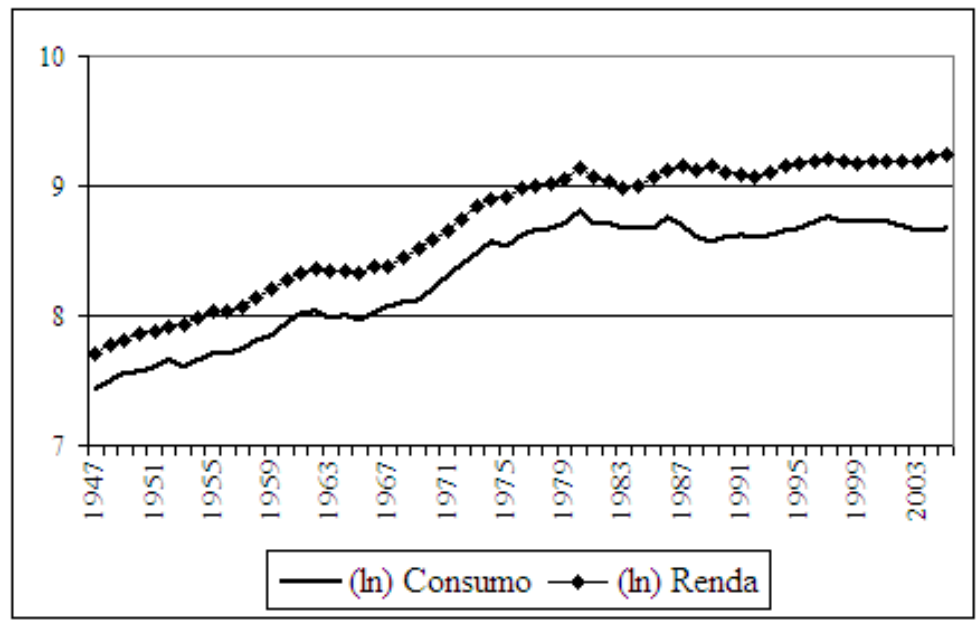

Figura 2: Juros real

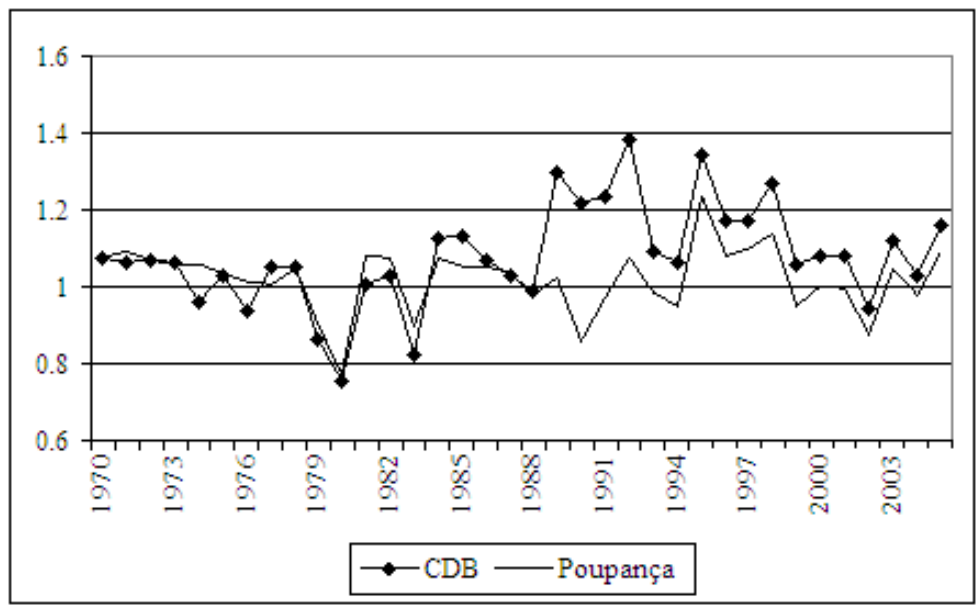


Como usual, foram utilizados testes de raiz unitária e, conforme reportado na Tabela 1, considerando o nível de significância de $5 \%$, os seguintes resultados são obtidos: $i$ ) os testes de Dickey and Fuller (1979) aumentado, ADF, e Phillips and Perron (1988), PP, rejeitam a hipótese nula de raiz unitária apenas para o CDB e a poupança; $i i$ ) o teste de Kwiatkowski et alii (1992), KPSS, rejeita a hipótese nula de estacionariedade apenas nos casos do consumo e da renda. Portanto, todos os testes sugerem que apenas o consumo e a renda são integrados. ${ }^{10}$

Tabela 1: Teste de raiz unitária

\begin{tabular}{lccc}
\hline Série & ADF & PP & KPSS \\
\hline (ln) Consumo & -0.510 & -0.661 & $0.217^{*}$ \\
(ln) Renda & -0.829 & -0.770 & $0.223^{*}$ \\
CDB & $-3.748^{*}$ & $-3.916^{*}$ & 0.338 \\
Poupança & $-5.323^{*}$ & $-5.332^{*}$ & 0.071 \\
\hline
\end{tabular}

Nota: Os testes para os consumo e renda consideram o período de 1947 a 2005 enquanto os testes para o CDB e a poupança consideram o período de 1970 a 2005 . A equação de teste inclui uma constante e no caso do consumo e da renda uma tendência linear foi adicionada. A hipótese nula do teste ADF e PP é a presença de raiz unitária enquanto a hipótese nula do teste KPSS é estacionariedade. * significa rejeição de $\mathrm{HO}$ a $5 \%$ e ** $10 \%$. O número de defasagens da variável independente incluídas na equação de teste do teste $\mathrm{ADF}$ foi definido com base no critério de Schwarz. O teste PP e KPSS usaram o núcleo de Bartlett e a janela de Newey-West.

Para estimar as equações (4) e (5) é utilizado o estimador de mínimos quadrados ordinários (MQO) e o estimador de mínimos quadrados em dois estágios (MQ2E). ${ }^{11}$ Neste caso, os instrumentos utilizados são defasagens das variáveis que aparecem na equação de teste a ser estimada. São utilizados três conjuntos diferentes de instrumentos: a primeira defasagem das variávies da equação de teste, a primeira e a segunda defasagens e da primeira à terceira defasagens. Para correção de possíveis problemas de heterocedasticidade e autocorrelação empregamos o estimador de Newey e West (1987) com núcleo de Bartllet e janela dada por $\left[4(T / 100)^{2 / 9}\right]$, em que $T$ é o tamanho da amostra. Os resultados são apresentados na próxima seção. Dada a maior disponibilidade de dados de consumo e renda, estima-se ainda as versões das equações (4) e (5) sem a inclusão do juros:

$$
\begin{gathered}
\Delta \ln \left(c_{t+1}\right)=\mu_{0}+\lambda \Delta \ln \left(y_{t+1}\right)+\varepsilon_{t+1} \\
\Delta \ln \left(c_{t+1}\right)=\pi_{0}+\pi_{1} I_{p} \Delta \ln \left(y_{t+1}\right)+\pi_{2} I_{n} \Delta \ln \left(y_{t+1}\right)+\varepsilon_{t+1}
\end{gathered}
$$

\subsection{Resultados econométricos}

Como a amostra não é muito grande, especialmente quando se considera a taxa de juros, nas análises subseqüentes é utilizado o nível de significância de $10 \%$ em todos os testes de hipótese. Na Tabela 2 , apresentamos os resultados para a equação (7). O coeficiente da renda é próximo de 1 quando se emprega o estimador de MQO e diminui com o uso do estimador de MQ2E, atingindo 0,859 quando são usados como instrumentos 3 defasagens das variáveis que aparecem na equação de teste. Em todos os modelos o coeficiente da renda é significativamente diferente de zero e o teste $J$ de sobreidentificação de Hansen não é rejeitado.

${ }^{10}$ Os mesmos testes de raiz unitária são aplicados à primeira diferença de $\ln \left(c_{t}\right)$ e $\ln \left(y_{t}\right)$. Os testes ADF e PP rejeitam a hipótese nula, ao nível de significância de $5 \%$, para as duas séries. O teste KPSS, não rejeita a hipótese nula de estacionariedade, ao nível de significância de $5 \%$, para as duas séries. Assim, a evidência sugere que $\ln \left(c_{t}\right)$ e $\ln \left(y_{t}\right)$ são $I(1)$.

${ }^{11}$ Ver nota 6. 
Tabela 2: Consumo e renda (equação 7)

\begin{tabular}{cccc}
\hline \multicolumn{4}{c}{ Variável dependente: $\triangle \ln \left(c_{t}\right) /$ Período: 1948 a 2005} \\
\hline $\begin{array}{c}\text { Coeficiente } \\
\text { intrumentos }\end{array}$ & $\begin{array}{c}\text { (erro padrão) } \\
\text { Defasagem dos }\left(y_{t}\right)\end{array}$ & $\begin{array}{c}\text { Teste } J \text { de Hansen } \\
\text { (p-valor) }\end{array}$ & $N$ \\
\hline- & $0.996^{*}$ & - & 58 \\
& $(0.126)$ & & \\
$1^{\mathrm{a}}$ & $0.961^{*}$ & 0.020 & 57 \\
& $(0.264)$ & $(0.888)$ & \\
$1^{\mathrm{a}}$ e $2^{\mathrm{a}}$ & $0.966^{*}$ & 1.140 & 56 \\
& $(0.250)$ & $(0.766)$ & \\
$1^{\mathrm{a}}, 2^{\mathrm{a}}$ e $3^{\mathrm{a}}$ & $0.859^{*}$ & 1.347 & 55 \\
& $(0.233)$ & $(0.930)$ & \\
\hline
\end{tabular}

Nota: Foi aplicado o estimador de MQ2E aliado ao estimador de Newey-West (1987) robusto a autocorrelação e heterocedasticidade. Os instrumentos são defasagens das variáveis que compõe a equação de teste, e o $\mathrm{n}^{0}$ de defasagens é reportado na primeira coluna. No primeiro caso, sem instrumento, é aplicado o estimador de MQO. Todos os modelos incluem uma constante não reportada. ${ }^{*}$ indica significância estatística a 10\%. A última coluna reporta o tamanho da amostra.

A Tabela 3 apresenta os resultados da equação (4). No caso do CDB, a estimativa de MQO para a renda de 0,958 é significativa enquanto o juros é não significativo. Aplicando o estimador de MQ2E, as estimativas para a renda são significativas, porém são muito sensíveis ao conjunto de instrumentos utilizados. Poucos instrumentos levam a um impacto maior da renda sobre o consumo, inclusive, com apenas uma defasagem como instrumento o coeficiente da renda superou o valor unitário. Ao aumentar o número de instrumentos, o coeficiente da renda diminui. Substituindo o CDB pela poupança, os resultados são similares, porém com uma defasagem apenas dos instrumentos a renda é não significativa. Em todas as estimações o juros (CDB ou poupança) é não significativo e o teste de $J$ de sobreidentificação não rejeita os modelos estimados. De um modo geral, os resultados da literatura nacional se repetem: a taxa de crescimento da renda tem um impacto elevado sobre a taxa de crescimento do consumo, enquanto o juros parece não ter papel algum.

Na Tabela 4, são apresentados os resultados para a equação (8), sendo investigado se o impacto da renda sobre o consumo depende de a renda ter sofrido um aumento ou uma redução. Usando o estimador de MQO, $I_{p} \Delta \ln \left(y_{t}\right)$ apresenta um coeficiente superior ao de $I_{n} \Delta \ln \left(y_{t}\right)$. Com a abordagem de variáveis instrumentais, este resultado se repete. Inclusive, o coeficiente de $I_{n} \Delta \ln \left(y_{t}\right)$ é sempre não significativo, o que é uma evidência à favor da existência de restrição de crédito. Ao aplicar um teste $F$ cuja hipótese nula é de que os coeficientes de $I_{n} \Delta \ln \left(y_{t}\right)$ e $I_{p} \Delta \ln \left(y_{t}\right)$ são iguais, não se rejeita esta hipótese, o que é uma evidência à favor de miopia. No entanto, sob miopia $I_{n} \Delta \ln \left(y_{t}\right)$ deveria ser significativo, o que não acontece. Neste sentido, a hipótese de miopia e restrição de crédito carecem ainda de uma comprovação empírica mais sólida, existindo evidências a favor e contra cada uma delas. Vale ressaltar que, em todos os casos, o modelo não é rejeitado pelo teste $J$ de sobreidentificação.

Por fim, na Tabela 5 são reportados os resultados para a equação (5). No caso do CDB, $I_{p} \Delta \ln \left(y_{t}\right)$ sempre apresenta coeficiente superior ao coeficiente de $I_{n} \Delta \ln \left(y_{t}\right)$ que é, mais uma vez, não significativa. Devem-se mencionar ainda dois resultados: usando 2 defasagens como instrumento, a taxa de juros é significativa e, usando 3 defasagens como instrumento, nenhum regressor foi significativo. Em todos os casos, não se rejeita a hipótese de que os coeficientes de $I_{n} \Delta \ln \left(y_{t}\right)$ e $I_{p} \Delta \ln \left(y_{t}\right)$ são iguais, no nível de significância de 5\%; porém, sob miopia, espera-se ainda que $I_{n} \Delta \ln \left(y_{t}\right)$ seja significativo, o que não ocorre. Por fim, o teste de $J$ de sobreidentificação não é rejeitado. Ao utilizar a poupança, o 
Tabela 3: Consumo, renda e juros, 1970-2005 (equação 4)

\begin{tabular}{|c|c|c|c|c|c|}
\hline \multicolumn{6}{|c|}{ Variável dependente: $\triangle \ln \left(c_{t}\right) /$ Período: 1970 a 2005} \\
\hline \multirow{3}{*}{$\begin{array}{l}\text { Série de } \\
\text { juros }\end{array}$} & \multirow{3}{*}{$\begin{array}{c}\text { Defasagem dos } \\
\text { instrumentos }\end{array}$} & \multicolumn{2}{|c|}{ Coeficientes } & \multirow{3}{*}{$\begin{array}{c}\text { Teste } J \text { de } \\
\text { Hansen } \\
\text { (p-valor) }\end{array}$} & \multirow{3}{*}{$N$} \\
\hline & & $\triangle \ln \left(y_{t}\right)$ & $r_{t}$ & & \\
\hline & & (erro padrão) & (erro padrão) & & \\
\hline \multirow{8}{*}{$\mathrm{CDB}$} & - & 0.958* & 0.005 & - & 36 \\
\hline & & $(0.149)$ & $(0.033)$ & & \\
\hline & $1^{a}$ & $1.292^{*}$ & 0.246 & 0.048 & 35 \\
\hline & & $(0.636)$ & $(0.182)$ & $(0.827)$ & \\
\hline & $1^{\mathrm{a}}$ e $2^{\mathrm{a}}$ & $0.884^{*}$ & 0.147 & 3.304 & 34 \\
\hline & & $(0.471)$ & $(0.090)$ & $(0.508)$ & \\
\hline & $1^{\mathrm{a}}, 2^{\mathrm{a}}$ e $3^{\mathrm{a}}$ & $0.662^{*}$ & -0.025 & 6.989 & 33 \\
\hline & & $(0.319)$ & $(0.057)$ & $(0.430)$ & \\
\hline \multirow{8}{*}{ Poupança } & - & 0.959* & -0.031 & - & 36 \\
\hline & & $(0.137)$ & $(0.067)$ & & \\
\hline & $1^{\mathrm{a}}$ & 1.940 & -0.661 & 0.354 & 35 \\
\hline & & (2.389) & (1.960) & $(0.552)$ & \\
\hline & $1^{a}$ e $2^{a}$ & $1.031^{*}$ & -0.073 & 1.637 & 34 \\
\hline & & $(0.367)$ & $(0.153)$ & $(0.802)$ & \\
\hline & $1^{\mathrm{a}}, 2^{\mathrm{a}}$ e $3^{\mathrm{a}}$ & $0.690^{*}$ & -0.035 & 5.233 & 33 \\
\hline & & $(0.325)$ & $(0.118)$ & $(0.632)$ & \\
\hline
\end{tabular}

Nota: Foi aplicado o estimador de MQ2E aliado ao estimador de Newey-West (1987) robusto a autocorrelação e heterocedasticidade. Os instrumentos são defasagens das variáveis que compõe a equação de teste, e o $\mathrm{n}^{\circ}$ de defasagens é reportado na primeira coluna. No primeiro caso, sem instrumento, é aplicado o estimador de MQO. Todos os modelos incluem uma constante não reportada. * indica significância estatística a 10\%. A última coluna reporta o tamanho da amostra.

estimador de MQ2E não obteve nenhum coeficiente significativo quando são utilizadas 1 defasagem ou 3 defasagens como instrumentos. No caso em que se utilizam 2 defasagens, $I_{p} \Delta \ln \left(y_{t}\right)$ e $I_{n} \Delta \ln \left(y_{t}\right)$ são significativos e o primeiro é menor do que o segundo. Porém, utilizando o teste $F$ não se rejeita a hipótese nula de igualdade, uma evidência favorável a hipótese de miopia.

Portanto, excetuando-se um único caso, observamos na Tabela 5 que a taxa de juros não é significativa, o que é uma evidência contra a hipótese de comportamento otimizador. Se os juros são irrelevantes, voltamos a Tabela 4, na qual não há uma resposta clara sobre o causa deste desvio do comportamento otimizador. Como mencionado, o coeficiente de $I_{p} \Delta \ln \left(y_{t}\right)$ é significativo e superior ao coeficiente de $I_{n} \Delta \ln \left(y_{t}\right)$, que é inclusive não significativo. Porém, o teste $F$ não rejeita a hipótese nula de simetria. Provavelmente, como uma das variáveis é não significativa, seu intervalo de confiança é bastante extenso, o que pode estar reduzindo o poder do teste $F$ para rejeitar a hipótese nula de igualdade entre os coeficientes. Nesta perspectiva, não há uma evidência clara à favor de miopia nem tão pouco de restrição de crédito.

Para aprofundar esta discussão, a Figura 3 apresenta $\Delta \ln \left(c_{t}\right)$ como função da $\Delta \ln \left(y_{t}\right)$. Quando $\Delta \ln \left(y_{t}\right)>0$, há uma associação positiva entre as taxas de crescimento do consumo e da renda. Porém, quando $\Delta \ln \left(y_{t}\right)<0$, não há qualquer relação evidente entre estas taxas de crescimento, o que explica a não significância de $I_{n} \Delta \ln \left(y_{t}\right)$. 
Tabela 4: Consumo e renda (equação 8)

\begin{tabular}{|c|c|c|c|c|c|}
\hline \multicolumn{6}{|c|}{ Variável dependente: $\triangle \ln \left(c_{t}\right) /$ Período: 1948 a 2005} \\
\hline \multirow{3}{*}{$\begin{array}{l}\text { Defasagem dos } \\
\text { instrumentos }\end{array}$} & \multicolumn{2}{|c|}{ Coeficientes } & \multirow{3}{*}{$\begin{array}{c}\text { Teste } F \text { de } \\
\text { igualdade } \\
\quad(p \text {-valor) }\end{array}$} & \multirow{3}{*}{$\begin{array}{c}\text { Teste } J \text { de } \\
\text { Hansen } \\
\text { (p-valor) }\end{array}$} & \multirow{3}{*}{$\begin{array}{l}N \\
(n) \\
\end{array}$} \\
\hline & $I_{p} \triangle \ln \left(y_{t}\right)$ & $I_{n} \triangle \ln \left(y_{t}\right)$ & & & \\
\hline & (erro padrão) & (erro padrão) & & & \\
\hline \multirow[t]{2}{*}{ - } & $1.178^{*}$ & 0.576 & 1.57 & - & 58 \\
\hline & $(0.155)$ & $(0.390)$ & $(0.215)$ & & (14) \\
\hline \multirow[t]{2}{*}{$1^{\mathrm{a}}$} & $1.193^{*}$ & -0.685 & 1.38 & 0.051 & 57 \\
\hline & $(0.460)$ & (1.296) & $(0.246)$ & $(0.821)$ & (14) \\
\hline \multirow[t]{2}{*}{$1^{\mathrm{a}}$ e $2^{\mathrm{a}}$} & $1.035^{*}$ & 0.705 & 0.22 & 3.648 & 56 \\
\hline & $(0.304)$ & (0.579) & $(0.642)$ & $(0.456)$ & (14) \\
\hline \multirow[t]{2}{*}{$1^{\mathrm{a}}, 2^{\mathrm{a}}$ e $3^{\mathrm{a}}$} & $0.968^{*}$ & 0.586 & 0.30 & 3.958 & 55 \\
\hline & $(0.307)$ & $(0.501)$ & $(0.585)$ & $(0.785)$ & (14) \\
\hline
\end{tabular}

Nota: Foi aplicado o estimador de MQ2E aliado ao estimador de Newey-West (1987) robusto a autocorrelação e heterocedasticidade. Os instrumentos são defasagens das

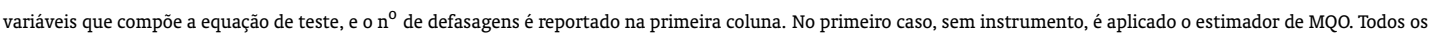
modelos incluem uma constante não reportada. *indica significância estatística a 10\%. A última coluna reporta o tamanho da amostra, $N$, bem como o número de períodos com variações negativas na renda, $n$.

Tabela 5: Consumo, renda e juros (equação 5)

\begin{tabular}{|c|c|c|c|c|c|c|c|}
\hline \multicolumn{8}{|c|}{ Variável dependente: $\triangle \ln \left(c_{t}\right) /$ Período: 1970 a 2005} \\
\hline \multirow{3}{*}{$\begin{array}{l}\text { Série de } \\
\text { juros }\end{array}$} & \multirow{3}{*}{$\begin{array}{c}\text { Defasagem dos } \\
\text { instrumentos }\end{array}$} & \multicolumn{2}{|c|}{ Coeficientes } & \multirow{3}{*}{$\begin{array}{c}\quad r_{t} \\
\text { (erro padrão) }\end{array}$} & \multirow{3}{*}{$\begin{array}{l}\text { Teste } F \text { de } \\
\text { igualdade } \\
\text { ( } p \text {-valor })\end{array}$} & \multirow{3}{*}{$\begin{array}{c}\text { Teste } J \text { de } \\
\text { Hansen } \\
(p \text {-valor) }\end{array}$} & \multirow{3}{*}{$\begin{array}{l}N \\
(n)\end{array}$} \\
\hline & & $I_{p} \triangle \ln \left(y_{t}\right)$ & $I_{n} \triangle \ln \left(y_{t}\right)$ & & & & \\
\hline & & (erro padrão) & (erro padrão) & & & & \\
\hline \multirow{8}{*}{$\mathrm{CDB}$} & \multirow[t]{2}{*}{-} & $1.247^{*}$ & 0.444 & 0.033 & 1.57 & \multirow[t]{2}{*}{-} & 36 \\
\hline & & $(0.217)$ & $(0.483)$ & $(0.040)$ & $(0.219)$ & & (11) \\
\hline & \multirow[t]{2}{*}{$1^{\mathrm{a}}$} & $1.742^{*}$ & -0.821 & 0.391 & 0.36 & 0.478 & 35 \\
\hline & & $(0.880)$ & (3.745) & $(0.255)$ & $(0.556)$ & $(0.489)$ & (11) \\
\hline & \multirow[t]{2}{*}{$1^{\mathrm{a}}$ e $2^{\mathrm{a}}$} & $1.333^{*}$ & 0.316 & $0.251^{*}$ & 0.64 & 3.969 & 34 \\
\hline & & $(0.683)$ & $(0.994)$ & $(0.139)$ & $(0.428)$ & $(0.554)$ & (11) \\
\hline & \multirow[t]{2}{*}{$1^{\mathrm{a}}, 2^{\mathrm{a}}$ e $3^{\mathrm{a}}$} & 0.953 & 0.073 & 0.035 & 0.51 & 8.239 & 33 \\
\hline & & $(0.585)$ & $(0.721)$ & $(0.071)$ & $(0.480)$ & $(0.510)$ & (11) \\
\hline \multirow{8}{*}{ Poupança } & \multirow[t]{2}{*}{-} & $1.179^{*}$ & 0.529 & -0.015 & 1.31 & \multirow[t]{2}{*}{-} & 36 \\
\hline & & $(0.197)$ & $(0.435)$ & $(0.064)$ & $(0.260)$ & & (11) \\
\hline & \multirow[t]{2}{*}{$1^{\mathrm{a}}$} & 0.021 & 2.206 & 0.656 & 0.29 & 0.152 & 35 \\
\hline & & (1.526) & (2.841) & $(0.807)$ & $(0.594)$ & $(0.697)$ & (11) \\
\hline & \multirow[t]{2}{*}{$1^{\mathrm{a}}$ e $2^{\mathrm{a}}$} & $0.965^{*}$ & $1.185^{*}$ & 0.091 & 0.08 & 5.461 & 34 \\
\hline & & $(0.388)$ & $(0.647)$ & $(0.208)$ & $(0.782)$ & $(0.362)$ & (11) \\
\hline & \multirow[t]{2}{*}{$1^{\mathrm{a}}, 2^{\mathrm{a}}$ e $3^{\mathrm{a}}$} & 0.569 & 0.409 & 0.105 & 0.03 & 8.899 & 33 \\
\hline & & $(0.537)$ & $(0.608)$ & $(0.152)$ & $(0.872)$ & $(0.447)$ & (11) \\
\hline
\end{tabular}

Nota: Foi aplicado o estimador de MQ2E aliado ao estimador de Newey-West (1987) robusto a autocorrelação e heterocedasticidade. Os instrumentos são defasagens das variáveis que compõe a equação de teste, e o ${ }^{\circ}$ de defasagens é reportado na primeira coluna. No primeiro caso, sem instrumento, é aplicado o estimador de MQO. Todos os modelos incluem uma constante não reportada. * indica significância estatística a 10\%. A última coluna reporta o tamanho da amostra, $N$, bem como o número de períodos com variações negativas na renda, $n$. 
Figura 3: Relação entre consumo e renda

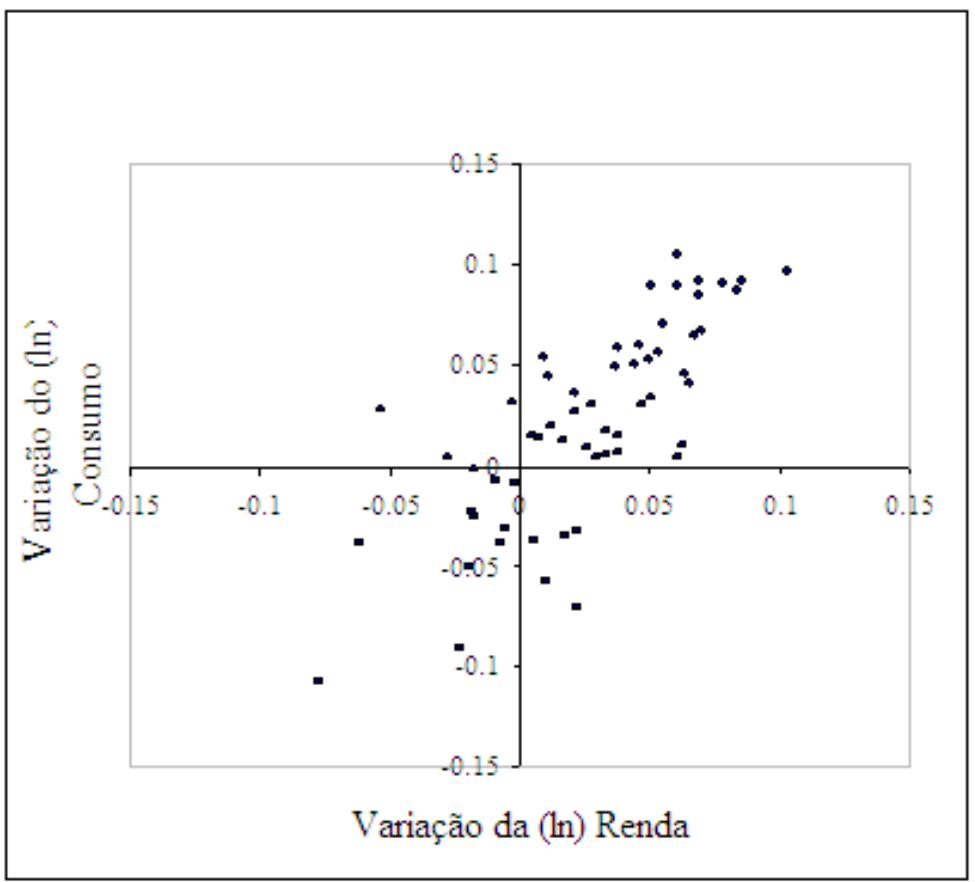

Analisando quadrante à quadrante, a primeira observação a ser feita é que a maior parte dos pontos concentra-se no $2^{\circ}$ quadrante, 41 observações (70,7\% da amostra), no qual as duas taxas de crescimento são positivas e a associação positiva entre elas é bastante evidente. No $4^{\circ}$ quadrante, no qual as duas variáveis são negativas, tem-se 9 observações (15,5\% da amostra). No $1^{\circ}$ e $3^{\circ}$ quadrantes $\Delta \ln \left(c_{t}\right)$ e $\Delta \ln \left(y_{t}\right)$ têm sinais opostos, o que é compatível com a hipótese de restrição de crédito, mas não com a hipótese de miopia. No $1^{\circ}$ quadrante, a poupança financia o consumo uma vez que a taxa de crescimento do consumo é positiva e a da renda é negativa. Desde que os agentes tenham recursos acumulados, isto é compatível com o cenário de restrição de crédito. No $3^{\circ}$ quadrante, ocorre o oposto, a renda cresce e o consumo cai. Este resultado pode ser devido a existência de um forte pessimismo quanto ao futuro que levaria os agentes restritos ao crédito a formarem uma espécie de poupança precaucional. A questão a ressaltar é que no $1^{\circ}$ e $3^{\circ}$ quadrantes, nos quais é possível distinguir a hipótese de miopia de restrição de crédito, existem apenas 8 observações ( $13,8 \%$ da amostra), o que pode ter contribuído para a incapacidade do modelo de distinguir estas duas hipóteses.

Os pontos do $1^{\circ}$ quadrante, nos quais há um crescimento do consumo mesmo com a retração da renda, referem-se aos anos de 1982, 1990 e 1991. Para ser preciso, em 1982 a taxa de crescimento do consumo é muito próxima de zero. Um resultado esperado, pois devido à queda do PIB em 1981 e a estagnação de 1982, provavelmente, não havia poupança suficiente para financiar um consumo maior. ${ }^{12}$

Já em 1990, imediatamente após a posse, Collor introduziu o programa antiinflacionário Collor I que, entre outras medidas, introduzia o Cruzeiro e congelava os depósitos overnight que excedessem a $N C z \$ 50$ mil, os salários e os preços, aumentava os preços dos serviços públicos, liberava o câmbio e

\footnotetext{
${ }^{12}$ Outra interpretação possível é que os agentes econômicos esperavam uma crise bastante longa e, por conseguinte, a suavização
} via poupança permitiu apenas manter o consumo praticamente inalterado. 
iniciava a abertura da economia à concorrência externa. O efeito líquido de todas estas medidas foi uma recessão. Como foi possível financiar um aumento do consumo, com o confisco de parte da poupança? 0 dinheiro bloqueado ficaria congelado por 18 meses e, posteriormente, seria devolvido em parcelas mensais, acrescidas de juros (Baer, 2002). No entanto, isto não ocorreu. Devido às pressões da sociedade, parte deste valor foi desbloqueado antes do prazo. Além disso, os agentes econômicos desenvolveram várias estratégias para burlar o congelamento. ${ }^{13}$ Tudo isto, permitiu um crescimento do consumo de quase $3 \%$ já em 1990. No mês de julho do ano seguinte, como reação ao retorno da inflação, foi implementado o Plano Collor II, que se baseava na eliminação do overnight, no congelamento de preços e salários e na extinção de várias formas de indexação (Baer, 2002). Porém, o governo não retroagiu no desbloqueio da poupança e, por fim, o consumo apresentou uma taxa de crescimento ligeiramente superior a $3 \%$. Vale ressaltar ainda que, a abertura econômica promovida pelo governo Collor, também potencializou o consumo na medida em que aumentou a concorrência e expôs o consumidor a novos bens e produtos.

Os pontos do $3^{\circ}$ quadrante referem-se aos anos de 1953, 1975, 1987, 1989 e 2002, nos quais apesar da renda apresentar uma taxa de crescimento positiva, o oposto ocorreu com o consumo. Como mencionado, uma explicação para este resultado seria um pessimismo por parte dos consumidores. No início de 1953, o Brasil vivenciou um colapso cambial e, como forma de combater a crise, o governo Vargas promulgou a Lei 1807, conhecida como Lei do Mercado Livre, sendo instituído o sistema de taxas múltiplas de câmbio e a liberdade de movimento pelo mercado livre de câmbio do capital estrangeiro. No entanto, havia a preocupação de que o novo sistema provocasse a desvalorização excessiva da taxa de câmbio livre, além de efeitos inflacionários. Paralelamente à incerteza econômica, as bases de sustentação do governo Vargas começam a se fragilizar pelas cobranças da classe operária das promessas suscitadas na campanha eleitoral e pelo avanço da oposição, que empolgava ao mesmo tempo a elite e o operariado, desgastando o governo federal (Vianna, 1990).

Em 1975 três fatos marcaram a condução da política econômica de curto prazo: a crise financeira, a queda do crescimento das exportações e a aprovação do II Plano Nacional de Desenvolvimento (Carneiro, 1990). O primeiro representou as dificuldades do governo Geisel em conter a inflação, em meio a crise externa provocada pelo choque do petróleo. O segundo colocava em risco as contas externas brasileiras (Carneiro, 1990). O terceiro consistia em um imenso programa de investimento com vistas a aumentar a auto-suficiência do país (Baer, 2002). Enfim, neste contexto de crise externa e implementação de um novo plano econômico, os agentes econômicos optaram por reduzir o consumo.

Segundo Carneiro e Modiano (1990) um dos fatores que marcaram o ano de 1987 foi o aumento da incerteza. Mesmo com a implementação do Plano Cruzado II, ao final de 1986, as taxas de inflação cresceram acentuadamente, atingindo $16,8 \%$ em janeiro de 1987 . Sem indícios de recuperação do saldo da balança comercial, que se tornara negativo, o governo declara moratória. Em junho de 1987, é anunciado o Plano Bresser que propunha uma estabilização híbrida, unindo elementos ortodoxos e heterodoxos como a indexação dos salários e o congelamento dos preços. Em janeiro de 1989, o Plano Verão promoveu uma nova reforma monetária, que, visando combater a inflação, pretendia contrair a demanda agregada a curto prazo. Porém, segundo Carneiro e Modiano (1990), a falta de credibilidade do plano, após o fracasso dos planos Cruzado e Bresser, fez com que seus objetivos não fossem alcançados e o seu insucesso conduziu a economia para um nível de indexação tão ou mais elevado do que antes do plano, ocorrendo inclusive um agravamento do desequilíbrio fiscal. Assim, "ressurgiu na sociedade brasileira o temor da hiperinflação" (Carneiro e Modiano, 1990, p. 381).

Por fim, em 2002, a economia brasileira passou por vários fatores causadores de incerteza, destacandose as fortes tensões oriundas de seu balanço de pagamentos (Baer, 2002); a recessão mundial, provocada pela crise argentina e pelo 11 de setembro; e, claro, as incertezas do cenário eleitoral. Neste contexto, é possível compreender porque o consumo se retraiu, apesar da elevação da renda.

\footnotetext{
${ }^{13}$ De fato, segundo dados do Ipeadata, o M1 (papel moeda em poder do público e depósitos à vista) cresceu cerca de $23 \%$ em 1990 .
} 
Concluindo, apesar de não podermos distinguir formalmente, através da análise econométrica, as hipóteses de miopia e restrição de crédito, há um indício de que em alguns momentos os consumidores suavizaram seu consumo, o que é incompatível com miopia. Assim, a explicação mais convincente para esta suavização não ocorrer de modo frequente, seria à falta de acesso ao crédito.

\section{CONCLUSÃO}

Este artigo confronta três teorias sobre a decisão de consumo a partir de dados da economia brasileira. A primeira supõe que os consumidores otimizam seu bem-estar intertemporalmente, através de uma Equação de Euler, cuja versão log-linearizada implica que a taxa de crescimento do consumo é uma função da taxa de juros. A segunda supõe que os consumidores são míopes e, por isso, consomem a sua renda corrente. A terceira supõe que os indivíduos não têm acesso a crédito, mas não há restrições ao acúmulo de riqueza e, como resultado, o consumo reagiria de forma assimétrica à renda, sendo afetado mais fortemente quando há aumentos na renda do que reduções.

Após as estimações, conclui-se que, em geral, os juros não são significativos, o que vai de encontro à hipótese de comportamento otimizador. No entanto, não é possível definir se este resultado é devido à hipótese de miopia ou restrição de crédito. No primeiro caso, espera-se que a renda tenha efeito simétrico sobre o consumo, o que não é rejeitado. Porém, o coeficiente da taxa de crescimento da renda, quando esta é negativa, é não significativo, uma evidência favorável à hipótese de falta de acesso ao crédito.

Com o intuito de elucidar esta questão, foram analisadas anualmente as taxas de crescimento do consumo e da renda. Em alguns anos estas taxas de crescimento apresentam sinais opostos, havendo alguma evidência ao encontro de suavização de consumo e de encontro à hipótese de miopia. Esta suavização é restrita à poucos anos, o que é compatível apenas com a hipótese de restrição de crédito. Portanto, há alguma evidência à favor desta hipótese.

\section{BIBLIOGRAFIA}

Altonji, J. G. \& Siow, A. (1987). Testing the response of consumption to income changes with (noisy) panel data. Quarterly Journal of Economics, 102:293-328.

Baer, W. (2002). A Economia Brasileira. Nobel, São Paulo, 2nd edition.

Bernanke, B. (1985). Adjustment costs, durable goods and aggregate consumption. Journal of Monetary Economics, 15:41-68.

Campbell, J. \& Mankiw, G. (1989). Consumption, income and interest rates: Reinterpreting the time series evidence. In Blanchard, O. J. \& Fischer, S., editors, National Bureau of Economic Research Macroeconomics Annual, pages 185-216. MIT Press, Cambridge, MA.

Carneiro, D. D. (1990). Crise e esperança: 1974-1980. In Abreu, M. P., editor, A Ordem do Progresso: Cem Anos de Política Econômica Republicana 1889-1989. Campus, Rio de Janeiro.

Carneiro, D. D. \& Modiano, E. (1990). Ajuste externo e desequilíbrio interno: 1985-1989. In Abreu, M. P., editor, A Ordem do Progresso: Cem Anos de Política Econômica Republicana 1889-1989. Campus, Rio de Janeiro.

Cavalcanti, C. B. (1993). Intertemporal substitution in consumption: An empirical investigation for Brazil. Brazilian Review of Econometrics, 13(2):203-229.

Dickey, D. A. \& Fuller, W. A. (1979). Distribution of the estimators for autoregressive time series with a unit root. Journal of the American Statistical Association, 74:427-431. 
Ellery Jr., R., Gomes, V., \& Sachsida, A. (2002). Business cycle fluctuations in Brazil. Revista Brasileira de Economia, 56(2):269-308.

Flavin, M. (1981). The adjustment of consumption to changing expectations about future income. Journal of Political Economy, 89(5):974-1009.

Friedman, M. (1957). A Theory of Consumption Function. Princeton University Press, Princeton, NJ.

Gomes, F. A. R. (2004). Consumo no Brasil: Teoria da renda permanente, formação de hábito e restrição à liquidez. Revista Brasileira de Economia, 58:381-402.

Gomes, F. A. R., Issler, J. V., \& Salvato, M. A. (2005). Principais características do consumo de duráveis no Brasil e testes de separabilidade entre duráveis e não-duráveis. Revista Brasileira de Economia, 59(1):33-60.

Gomes, F. A. R. \& Paz, L. S. (2004). Especificações para a função consumo: Testes para países da América do Sul. Pesquisa e Planejamento Econômico, 34(1):39-55.

Hall, R. E. (1978). Stochastic implications of the life cycle permanent income hypothesis: Theory and evidence. Journal of Political Economy, 86:971-87.

Hall, R. E. (1988). Intertemporal substitution in consumption. Journal of Political Economy, 96:339-357.

Hansen, L. P. \& Singleton, K. J. (1983). Stochastic consumption, risk aversion andthe temporal behavior of asset returns. Journal of Political Economy, 91:249-265.

Issler, J. V. \& Rocha, F. P. (2000). Consumo, restrição à liquidez e bem-estar no Brasil. Economia Aplicada, 4(4):637-665.

Kwiatkowski, D., Phillips, P. C. B., Schmidt, P., \& Shin, Y. (1992). Testing the null hypothesis of stationary against the alternative of a unit root. Journal of Econometrics, 54:159-178.

Mankiw, N. G. (1981). The permanent income hypothesis and the real interest rate. Economics Letters, 7:307-311.

Mankiw, N. G. (1982). Hall's consumption hypothesis and durable goods. Journal of Monetary Economics, 10:417-425.

Newey, W. \& West, K. (1987). A simple positive semi-definite, heteroskedasticity and auto-correlation consistent covariance matrix. Econometrica, 55:703-708.

Phillips, P. C. B. \& Perron, P. (1988). Testing for a unit root in time series regression. Biometrika, 75:335346.

Reis, E., Issler, J. V., Blanco, F., \& Carvalho, L. (1998). Renda permanente e poupança precaucional: Evidências empíricas para o Brasil no passado recente. Pesquisa e Planejamento Econômico, 28(2):233272.

Shea, J. (1995). Myopia, liquidity constraints and aggregate consumption: A simple test. Journal of Money, Credit \& Banking, 27:798-805.

Vianna, S. B. (1990). Duas tentativas de estabilização: 1951-1954. In Abreu, M. P., editor, A Ordem do Progresso: Cem Anos de Política Econômica Republicana 1889-1989. Campus, Rio de Janeiro. 


\section{A. APÊNDICE}

Considere a Equação de Euler:

$$
E_{t}\left[\left(\frac{c_{t+1}}{c_{t}}\right)^{-\sigma}\left(1+r_{t+1}\right)\right]=\frac{1}{\beta}
$$

Seja $g_{t+1} \equiv c_{t+1} / c_{t}-1$ a taxa de crescimento do consumo. A expansão de Taylor de primeira ordem ao redor de $r^{*}=g^{*}=0$ de $f(r, g)=(1+g)^{-\sigma}(1+r)$ é dada por

$$
\begin{aligned}
f(r, g) & \left.\simeq f(r, g)\right|_{r^{*}=g^{*}=0}+\left.\frac{\partial f(r, g)}{\partial r}\right|_{r^{*}=g^{*}=0} r+\left.\frac{\partial f(r, g)}{\partial g}\right|_{r^{*}=g^{*}=0} g \\
& =\left.(1+g)^{-\sigma}(1+r)\right|_{r^{*}=g^{*}=0}+\left.(1+g)^{-\sigma}\right|_{r^{*}=g^{*}=0} r-\left.\sigma(1+g)^{-\sigma-1}(1+r)\right|_{r^{*}=g^{*}=0} g \\
& =1+r-\sigma g
\end{aligned}
$$

Por conseguinte,

$$
E_{t}\left[\left(1+g_{t+1}\right)^{-\sigma}\left(1+r_{t+1}\right)\right] \approx E_{t}\left[1+r_{t+1}-\sigma g_{t+1}\right]
$$

E,

$$
1+r_{t+1}-\sigma g_{t+1}+v_{t+1}=\frac{1}{\beta}
$$

em que $E_{t}\left(v_{t+1}\right)=0$. Finalmente,

$$
\begin{aligned}
g_{t+1} & =-\frac{1}{\sigma} \frac{1-\beta}{\beta}+\frac{1}{\sigma} r_{t+1}+\frac{1}{\sigma} v_{t+1} \\
\frac{c_{t+1}}{c_{t}}-1 & =\mu+\frac{1}{\sigma} r_{t+1}+\varepsilon_{t+1}
\end{aligned}
$$

em que $\mu=-(1-\beta) / \sigma \beta, \varepsilon_{t+1}=v_{t+1} / \sigma$ e, por conseguinte, $E_{t}\left(\varepsilon_{t+1}\right)=0$. Como, $\ln (1+z) \approx z$ para $z$ pequeno, é comum estimar

$$
\Delta \ln \left(c_{t+1}\right)=\mu+\frac{1}{\sigma} r_{t+1}+\varepsilon_{t+1}
$$

